\title{
Buchbesprechungen - Book Reviews - Livres Nouveaux
}

Candardjís, G.: Diagnostic différentiel radiologiques des ulcerations gastriques.

Masson \& Cie., Paris 1956. 187 pages, 92 fig.

Depuis les travaux de Gutmann, le diagnostic de la nature, bénigne ou maligne, d'un ulcère gastrique, repose presque exclusivement sur $\Gamma$ analyse des cliches radiologiques; et malgré tous les perfectionnements techniques survenus depuis 20 ans, c'est finalement Tinterprétation des images de contraste baryté qui guideront la main du chirurgien, plus même que ses constatations visuelles ou tactiles au cours de Гopération.

On conçoit done toute $\Gamma$ importance du «diagnostic différentiel radiologique des ulcerations gastriques», dont Гinterprétation a donné lieu à tant de débats et dont C. a tenté la mise au point; et «il a fait preuve, en écrivant ce livre, de courage et d'application», comme Гaffirme P. Porcher dans son excellente preface; «De courage dans le choix du sujet, qui est épineux entre tous... d'application dans la lecture approfondie et critique de $\Gamma$ immense littérature, qui sous des angles et des tendances différentes, aborde son sujet.» La premiere partie est consacrée - après un paragraphe de technique - à la sêmíohgíe radiologique, et réussit à préciser la signification des divers aspects si âprement discutés en France.

Buchbesprechungen - Book Reviews - Livres Nouveaux 127

La deuxième partie, qui représente les trois-quarts du volume, comporte Гana-lyse systématique de documents provenant des Services universitaires de Lausanne, en s'appuyant sur la seule base solide - bien que contestée par certains -: Гexamen histologique des estomacs réséqués.

C. a revu les dossiers de 133 ulcères gastriques opérés entre 1950 et 1954, sur lesquels 16 étaient cancérisés et 9 des cancers ulcériformes. Et $\Gamma$ étude des cliches lui permet des conclusions precises. Tout d'abord, Гaffirmation qu'à condition d'individualiser une technique radiologique parfaite, il est souvent possible de poser un diagnostic de nature dès le premier transit baryté. Cela enlève déjà de Гintérêt au traitement d'épreuve, considéré comme le pilier du diagnostic «radio-clinique» de $\Gamma$ ulcère suspect; $\mathrm{C}$. va plus loin, et soutient que $\Gamma$ «on n'est en droit d'attendre, que si Гon a affaire à un syndrome radiologique typique de niche bénigne sur la portion verticale», adoptant ainsi une attitude résolument interventionniste.

Ce n'est pas le lieu ici d'exposer comment Гauteur s'est efforcé, tout au long de chaque chapitre, de justifier ces conclusions: mais ce point de vue aussi catégorique qu'original doit engager tous ceux qui cherchent une orientation plus precise dans ce diagnostic différentiel si difficile, à assimiler soigneusement le contenu de ce volume. M. Demole, Geneve Goldhahn, R., und G. Jorns: Lehrbuch der speziellen Chirurgie, Bd. II. VEB Georg Thieme Verlag, Leipzig 1956. XV + 608 Seiten, DM 66.--

Der vorliegende 2. Teil des überall günstig aufgenommenen Lehrbuches aus der deutschen demokratischen Republik umfaßt die Kapitel Bauch, Harn- und Ge-schlechtsorgane sowie Gliedmaßen. 
Jedem Abschnitt geht wiederum eine anatomisch-physiologische Betrachtung, erläutert mit zum Teil farbigen Skizzen, voraus. Die Indikationen und Möglich-keiten der modernen Magenchirurgie werden anschaulich und sehr verständlich beschrieben. Die Zunahme der Malignome des Dickdarmes rechtfertigt auch einen Einblick in die Methoden dieser operativen Behandlung. Differentialdiagnostische Tabellen erleichtern das Verständnis der verschiedenen Formen von Ileus. Daß Appendizitis auch für den Erfahrenen eine der schwierigsten Diagnosen ist, geht auch aus der klassischen Beschreibung dieses Buches hervor. Überzeugend wird der Frühoperation des Gallensteinleidens das Wort geredet. Die viel diskutierte akute und chronische Pankreatitis betrachtet der Chirurg heute noch als seine Domäne und ist daher berechtigt, aus seiner Erfahrung darüber zu berichten.

Die Verfasser scheinen mit den allgemeinen Behandlungsgrundsätzen der deutschen Urologen sehr gut vertraut zu sein. Auf kunstgerechte Katheterisierung wird größter Wert gelegt. Dieser kleine Eingriff, den jeder Arzt beherrschen soil, wird, nicht umsonst bis in alle Einzelheiten beschrieben. Die Überalterung der Bevölke-rung bringt die Ärzteschaft auch mit dem Prostataleiden immer mehr in Berührung. Wann und wie operiert wird, soil daher jeder wissen. Die Frakturenlehre lehnt sich eng an das Standardwerk von Böhler an. Die heute vielerorts geübte Osteosynthese wird nur für ausgewählte Spezialfälle emp-fohlen. Die Möglichkeiten der Hautplastik werden gestreift. Eine Abhandlung über Gelenkleiden, unter denen die degenerativen Formen einen immer größern Platz einnehmen, beschließt das Buch.

128 Buchbesprechungen - Book Reviews - Livres Nouveaux In seiner Einfachheit und Klarheit, mit den zahlreichen differentialdiagnostischen Tabellen, den ausgewählten Operationsskizzen und den farbigen anatomischen Tafeln hat das Lehrbuch einen hohen didaktischen Wert.

Jeder Arzt ist in der Lage, sich in dem Werk rasch und knapp über alles Wissens-werte der modernen Chirurgie ins Bild zu versetzen. Die einzelne Abhandlung liest sich mühelos und fließend. Diese Eigenschaften machen das Werk insbesondere zum handlichen Ratgeber des Studierenden und des Allgemeinpraktikers. Die chirurgischen Abdominalerkrankungen sind aber auch für den Gastro-Enterologen erschöpfend dargestellt.

Auf Literaturhinweise wurde verzichtet.

Das Buch halt sich streng an medizinische Erkenntnisse. Bemerkungen ideologischer Art sind vermieden, so daß es jedem Schweizer Arzt vorbehaltlos empfohlen werden kann. Hans Studer, Basel

Seífert, G.: Die Pathologie des kindlichen Pankreas. VEB Georg Thieme Verlag, Leipzig 1956, 151 Seiten, 109 Abb., davon 21 farbig. DM. 52.-.

Die vorliegende monographische Bearbeitung der Orthologie und Pathologie des kindlichen Pankreas gründet sich auf 506 Sektionsfälle (183 Frühgeburten, 262 Säuglinge bis zum 12. Lebensmonat und 61 Kinder bis zum 15. Lebensjahr), die in einem Zeitraum von 4 Jahren am Pathologischen Institut der Universität Leipzig gesammelt und bearbeitet wurden. Der Verf. sieht seine Aufgabe insbesondere darin, «das morphologische Substrat zur Physiologie im

Lebensablauf und zur pathologischen Physiologie der Krankheitsbilder in Beziehung zu setzen». Nach einleitender Besprechung der Entwicklungsgeschichte, der normalen Ana-tomie, der Zytologie, der Physiologie und des Wachstums folgt ein Kapitel über die allgemeine Pathologie des Pankreas. Hier werden die für die Struktur des Pankreas typischen morphologischen Reaktionsmuster des exkretorischen Teiles des Insel-apparates und des Mesenchyms herausgearbeitet. Unter dem Sammelbegriff der Dyschylie werden alle morphologisch erkennbaren Sekretionsstörungen unter Einschluß der Fermententgleisung zusammengefaßt, und 
der Verf. unternimmt den Versuch, die nicht entzündlichen Pankreasveränderungen (Dyschylie und Pankreasnekrose) von der Pankreatitis abzugrenzen.

Der spezielle Teil von etwa 80 Seiten enthält unter weitgehender Berücksichti-gung klinischer Fragestellungen eine ausgezeichnete Beschreibung der großen Krankheitsbilder: der Entwicklungsstörungen, der zystischen Pankreasfibrose, der entzündlichen Veränderungen, der Pankreasgeschwülste und der Mitbeteiligung des Pankreas bei Infektionen und Systemerkrankungen. Der Pathologie des Insel-systems ist ein eigenes Kapitel gewidmet, in dem die Tumoren, der kindliche Diabetes und die Embryopathia diabetica besprochen werden. Den Abschluß des Werkes bildet eine Abschnitt über die Syntropie der Veränderungen am Pankreas und an den Mundspeicheldrüsen mit 465 selbst untersuchten Fallen. Die Weltliteratur ist in einem Verzeichnis von 23 Seiten vollständig erfaßt.

Die Darstellung, das teilweise farbige Bildmaterial und die buchtechnische Ausstattung sind hervorragend. Die mustergültige Monographie muß als ein Mark-stein der Pankreas-Pathologie bezeichnet werden und wird Kliniker und Patho-logen über lange Zeit Anregung zur "Weiterarbeit geben können.

J. Línzbach, Marburg

Corrigenda

In der Arbeit

Über die Versorgung der Magen $\beta$ chleimhautzellen mit wasserlöslicheđ Gaßen Von L. DEMLING, R. GROMOTKA und N. HENNING

aus Vol. 85, Nr. 5/6, Seite 306fГ. dieser Zeitschrift ßînd durch eín bedauerliches Ver-sehen die Abbildungen fortgelassen worden, die wir im Folgenden wiedergeben:

Abb. 1. Kaninchenversuch; beim Verschluß der Art. coeliaca in der 7. Minute $\beta$ inkt die Azetylenausscheidung in den Magen rasch ab und steigt nach Eröfi $1 / 8$ ung der

Klemme wieder an.

\section{; $35797 / / 315 \quad 20 \quad 25 \mathrm{Mm} .30$}

Abb. 2. Mittelwerte der Azetylenausscheidung bei 15 normaciden Magengesunden.

9 Gastroenterologia, Vol. 86, No. 2 (1956)

130

Corrigenda

$0,5-1 \mathrm{Q} 4 \cdot 0,3-$

0,20, !

$\mathrm{Ui}$

$/ 35791 ! 1315$

20

$25 \mathrm{Mm} .30$

Abb. 3. Mittelwerte der Azetyleiiausscheidung bei 13 Patienten mit Superacidität. Flacher Kurvenverlauf, spates Maximum.

Abb. 4. Mittelwerte der Azetylenausscheidungskurven bei 14 Patienten mit Anacidität und bioptisch gesicherter Atrophie der Magenschleimhaut. Die gestrichelte Kurve zeigt vergleichsweise den Mittelwert normaler Kurvenverläufe. 


\section{Corrigenda}

131

15 Win. 30

I $3579 \prod 1315$

Abb. 5. Kurvenverläufe bei anfänglich florider, später unter klinischer Behandlung abklingender Oberflächengastritis mit beginnender Atrophie (bioptisch gesichert).

ó

9 .

$97 \mathrm{~h}$

$1 \cdot$

mc $1 / 82$

I

$/ 35 \mathrm{M}, \mathrm{n}$

\section{7}

Abb. 6. Häufigkeit der Kurvenmaxima in Abhängigkeit von der Zeit. I: Patienten mit Anacidität und Magenschleimhautatrophie (Mittelwert der Maxima: E=0,87). II: Gesunde normacide Probanden (Mittelwert der Maxima: E =0,43). Ill: Patienten mit Superacidität (Mittelwert der Maxima: $\mathrm{E}=0,27)$.

Corrigenda

:urven

$8-$

.1466. 7. Die gestrichelte Kurve zeigt die Mittelwerte von 11 Ausscheidungsk_ vor Antrenylgabe. Die ausgezogene Kurve stellt den Mittel·wert der Ausscheidung kurven 15 Minuten nach Antrenyl (1 mg s. c.) dar.

I 3579 H 13 ï5

$20 \quad 25 \mathrm{l} \cdot \mathrm{fm} 3 \hat{\mathrm{u}}$

Abb. 8. Zunahme der Azetylenausscheidung 15 Minuten nach Gabe von 1 mg Mesti-non s. c. (gestrichelte Kurve).

Abb. 9. Reduktion der Azetylenausscheidung 15 Minuten nach 0,05 g Ephetonin s.c. (gestrichelte Kurve).

Editor's Note

This is the first of the three consecutive numbers of Gastro-enterologia to be devoted to the papers and discussions heard at the Congress. The numbers are later to be bound together to constitute the Proceedings, a copy of which will be sent to each delegate.

Harold C. Edwards, 152, Harley Street, London, W. 1.

Discussion

Tríadu, I. (España) (74) 206

Malm, A. (Sweden) $\quad$ (76) 208

Rocha, A. (Spain) (78) 210

Roubícek, J. (Czechoslovakia)

(79) 211

c) Radiological Studies 
Koch, W. E., y M.-J. M. Corcuera (Chile): Estudio clinico radiologico en Disfagias por alteraciones Faringo-Esofagicas

(80) 212

Nauta, J. (Holland): The Closing Mechanism between the Oesophagus and the Stomach (87) 219

Monges, H. (France): Considerations sur le role du diaphragme dans la physio-logie de la continence gastro-cesophagienne et sur la projection radiologique

de Thiatus æsophagien

(100) 232

Nemours-Auguste, A. (France): Radio-physiologie de Гextrémité inférieure de Гæsophage (110) 242

Brombart, M. (Belgique): Radiologie des affections non-cancéreuses de Гæso phage (125) 257

Discussion

Banche, M., V. Lombardo e D. Fíandesío (Italia)jj

d) Clinical Aspects of Incompetence of the Cardía

(134) 266

Wínkelsteín, A. (U.S.A.): Peptic Esophagitis, Marginal Ulceration and Peptic Ulcer of the Esophagus. Recent Concepts (136) 268

Hillemand, P. (France): A propos du reflux æsophagien (140) 272

Allison, P. R. (Great Britain): Surgery of Non-Malignant Oesophageal Ste nosis (151) 283

Dunlop, E. E. (Australia): Problems in the Treatment of Reflux Oesophagitis Belsey, R. H. R. (Great Britain): Oesophageal Obstruction in Childhood. ...

Valdoní, P., and G. Marcozzí (Italy): Peptic Esophagitis

(172) 304

(155) 287

(169) 301

Discussion

Plocker, L., et M. É́lbaum (Pologne) (176) 308

Begley, B. C. (U.S.A.)

(179) 311

Boon, T. H. (Great Britain) (183) 315

Seríñana, J. Ma. (España) (185) 317

Gill, A. M. (Great Britain)

(187) 319

PREMALIGNANT CONDITIONS OF THE ALIMENTARY TRACT

Doll, R. (Great Britain): Environmental Factors in the Aetiology of Cancer

of the Stomach (188) 320

Bonadíes, A. (Italie): Les lesions précancéreuses de Tappareil digestif

Dukes, C. E. (Great Britain): Pathology of Precancerous Lesions of the Colon and Rectum (204) 336

Galambos, J. T., B. W. Massey, M. I. Klayman and J. B. Kírsner (U.S.A.):

Exfoliative Cytology in Chronic Ulcerative Colitis (206) 338

Gutmann, R. A., et G. Albot (France): Les lesions précancéreuses gastriques

Morson, B. C. (Great Britain): Intestinal Metaplasia of the Gastric Mucosa (221) 353

Berg, H. H. (Deutschland): Über die Entdeckung präkanzeröser Veränderungen am Magen (224) 356

Kapp, H. (Schweiz): Klinische Bedeutung der Präkanzerosen des Magens . .

Hess, R. (Switzerland): Early Cancer of the Stomach

(233) 365

Discussion

Rutkowskí, J., and St. Adamski (Poland)

(238) 370

Hess, W. (Schweiz) (240) 372

Felcí, L. (Italy) (242) 374 
Sullivan, W. A. Jr., A. P. Thai and 0. H. Wangensteen (U.S.A.)

(243) 375

Macchíoro, G. (Italia) (244) 376

Krondl, A., J. Knobloch and J. Fíklícký (Czechoslovakia)

(246) 378 